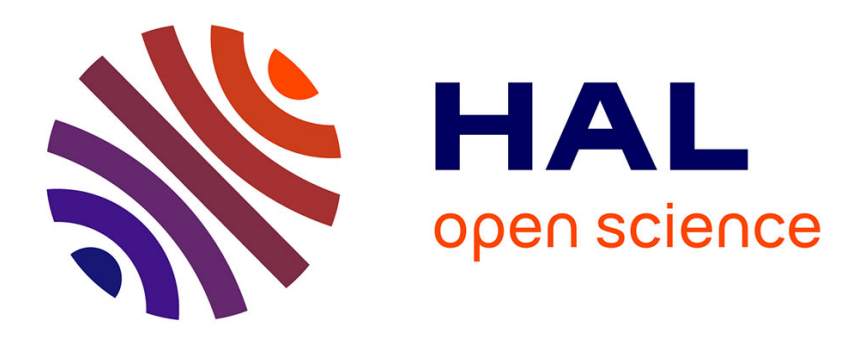

\title{
Charge ordering and scattering pre-peaks in ionic liquids and alcohols
}

Aurélien Perera

\section{To cite this version:}

Aurélien Perera. Charge ordering and scattering pre-peaks in ionic liquids and alcohols. Physical Chemistry Chemical Physics, 2017, 19 (2), pp.1062 - 1073. 10.1039/C6CP07834F . hal-01430812

\section{HAL Id: hal-01430812 \\ https://hal.sorbonne-universite.fr/hal-01430812}

Submitted on 10 Jan 2017

HAL is a multi-disciplinary open access archive for the deposit and dissemination of scientific research documents, whether they are published or not. The documents may come from teaching and research institutions in France or abroad, or from public or private research centers.
L'archive ouverte pluridisciplinaire HAL, est destinée au dépôt et à la diffusion de documents scientifiques de niveau recherche, publiés ou non, émanant des établissements d'enseignement et de recherche français ou étrangers, des laboratoires publics ou privés. 


\title{
Charge ordering and scattering pre-peak in ionic liquids and alcohols
}

\begin{abstract}
Aurélien Perera
Laboratoire de Physique Théorique de la Matière Condensée (UMR CNRS

7600), Université Pierre et Marie Curie, 4 Place Jussieu, F75252, Paris cedex 05, France.

Abstract

The structural properties of ionic liquids and alcohols are viewed under the charge ordering process as a common basis to explain the peculiarity of their radiation scattering properties, namely the existence, or absence, of a scattering pre-peak. Through the analysis of models, it is shown that the presence, or absence, of a radiation scattering pre-peak is principally related to the symmetry breaking, or not, of the global charge order, induced by the peculiarities of the molecular shapes. This symmetry breaking is achieved, in practice, by the emergence of specific types of clusters, which manifest how global charge order has changed into a local form. The various atomatom correlations witness the symmetry breaking induced by this re organization, and this is manifested into a pre-peak in the structure factor. This approach explains why associated liquids such as water do not show a scattering pre-peak. It also explains under which conditions core-soft models can mimic associating liquids.
\end{abstract}

\section{Introduction}

While liquids are fundamentally disordered systems, the presence of partial charges in constitutive molecules imposes a special form of global order, namely the charge order induced by the fact that charges of opposite valence attract each other, while those of same valence repel each other.

The simplest systems to show charge order are molten salts, and the existence of a charge ordering in these system has been studied in the mid seventies[1, 2, 3]. Charged ordering in electrolytes is less apparent, principally due to the low 
dilutions, as well as the strong ordering of the polar solvent near the anions and cations, which screens their relative charge ordering tendencies[4]. Recently, the study of room temperature ionic liquids have attracted a renewed attention on charge ordering, principally in order to explain the existence of a scattering prepeak in such liquids $[5,6,7,8,9]$. However, this analysis is biased by the inherent complexity of the molecules that make up such complex liquids. Indeed, such molecules have many charged atoms or groups in addition to neutral ones, and it is difficult to distinguish separately the contributions of every group in the global molecular distribution[10, 11].

Another class of liquids, namely alcohols, is equally known to exhibit scattering pre-peaks since early scattering investigations[12, 13, 14, 15, 16, 17]. From physical chemistry point of view these two categories of liquids belong to entirely different systems, with very different properties. However, the nature of their molecular order share the same common basis, namely charge ordering of the atomic groups. This is the point of view adopted in the present paper. In the classical representation of molecular liquids, various atoms are endowed with partial charges, resulting in a net charge for molecules making up ionic liquids and zero charge for neutral ones. However, it is the Coulomb interaction that dominates the way the molecules in each of these systems order themselves in the liquid state. It is this simple fact which allows to unify very different types of liquids from the same local manifestation principle, namely charge ordering.

From this point of view, several features common to both types of systems can be noticed, leading to a unifying point of view. Molten salts are endowed with the simplest form of charge ordering, which is global. In contrast, room temperature ionic liquids witness their charge ordering considerably perturbed by the existence of neutral groups and various type of charged sites on the same molecule. In that, they are similar to alcohols, whose molecules share similar characteristics (at the exception of being globally neutral). In this context of classical modeling through Coulomb interactions, both the phenomenon of Coulomb association and hydrogen bond interactions are treated in the same footing of Coulomb pairing of opposite charges. The existence of neutral groups in molecules lead to a disturbance in the homogeneity and the isotropy of charge distribution, as reflected by the global charge ordering. In order to comply to the constraint of preserving charge order despite the presence of neutral groups, charge order is reduced to a local manifestation -instead of the global one without these neutral groups. This is similar to a symmetry breaking transformation, in a physical sense rather than the usual mathematical sense used in Physics[18, 19]. It leads to marked changes in the correlation functions, which manifest themselves as a pre-peak in the corresponding partial structure factors. This mechanism is common to both ionic liquids and alcohols. This is the first principal message of the present paper.

The present study is essentially conducted by analysing the various atom-atom 
structure factors, as obtained by integral equations and computer simulations[20], and the total scattering function is rebuilt from these partial structure factors in order to be compared with that obtained through small or wide angle Xray scattering (SAXS or WAXS) experiments[5, 6, 21]. The second message of this paper is that radiation scattering experiments have appealing analogy with the BhatiaThornton (BT) transformation[22]. This analogy explains why global charge order is not seen in the total scattering function, and only local charge order is seen as a pre-peak. The absence of neutral groups in molecules such as water suffice to explain why global charge order is not broken, hence no pre-peak appears in the partial structure factors.

A third message of this paper is that the long range nature of the Coulomb interaction is irrelevant for charge order: it is only the magnitude of this interaction which is important. Therefore screened Coulomb interactions, which suppress the $1 / r$ singularity, equally qualify for charge order. This is perhaps in variance with previous works which have focused on the fact that the Coulomb interaction is one of the fundamental interactions in Nature, as well its particular mathematical properties due to the $1 / \mathrm{r}$ form. This is the case in earlier statistical theory of charged liquids[2,3]. For example, the landmark review by Baus and Hansen[3] cites charge ordering only once.

In view of the local molecular arrangements induced by charge ordering, another topic comes into light, that of the density fluctuations. Density fluctuations are also about local fluctuations in the distribution of the molecules. In what such fluctuations are different from charge ordering? Our answer to this question follows from our previous work[23]. Both phenomena are related to fluctuations, but at different scales. Density fluctuations are thermodynamical quantities (related to compressibility) and concern zero wave vector part of the response function. Charge order concern a specific non-zero wave vector, which correspond to a precise spatial scale over which local order occurs. In the present paper we expect to further clarify this issue.

The remainder of this paper is as follows. In the next section, we discuss the nature of charge order and illustrate it in the simplest case of charged spheres and what is its relation with Coulomb interaction. In the 3rd section, we will examine the cases of ionic liquids in general and alcohols in the context of charge ordering. The last section gathers discussion about the role of charge order in a more general context, as well as our conclusions about this study.

\section{Charge order}

It is instructive to examine the nature of the interactions, correlations and associated fluctuations in an equimolar binary mixtures of species $\mathrm{A}$ and $\mathrm{B}$, in the dense 
liquid region. If like species attract each other and $\mathrm{A}$ and $\mathrm{B}$ repel each other, both liquids might mix at high temperature, but there will be a threshold temperature below which the like attractive interaction will overcome the repulsive one and the mixture will undergo a macroscopic phase transition. Just above this transition, the mixture will experience large concentration fluctuations. Now, in the case of charged species $\mathrm{A}(+)$ and $\mathrm{B}(-)$, the interactions are exactly dual of the previous case: like particles repel each other and $\mathrm{A}$ and $\mathrm{B}$ attract each other. This mixture will never undergo phase separation. In fact, it will tend to be homogeneously mixed, with a "near" perfect alternation of A and B particles, since perfect alternation can be achieved only in a crystal of ionic salt. The impossibility of critical demixing concentration fluctuation is a remarkable character of charge ordered system, and this manifests itself into charge order. We note that the absence of demixing does not rule out the possibility if a liquid-gas phase separation at low temperatures, which is well documented[24, 25, 26, 27, 28, 29].

In this section, we use integral equation techniques, which consist in numerically solving jointly the Ornstein-Zernike equation with the hypernetted-chain closure, in order to obtain the correlation functions[20]. In Ref.[23], we have shown that such techniques are accurately in par with computer simulation for charged systems, as far as structural properties are concerned.

\subsection{A simple model of charge order}

To illustrate the considerations above, we consider a model of charged soft spheres $\mathrm{A}$ and $\mathrm{B}$, with valences $z_{A}=\left|z_{B}\right|=z, z_{A}>0$ and $z_{B}<0$, which are identical in diameter $\sigma$ and interact through the interaction consisting in a repulsive soft sphere and a screened Coulomb interaction. The general type of interaction between two particles we consider in this work is of the type:

$$
v_{a b}(r)=4 \varepsilon\left[\left(\frac{\sigma}{r}\right)^{12}-\lambda\left(\frac{\sigma}{r}\right)^{6}\right]+L_{B} \frac{z_{a} z_{b}}{r} \exp (-r / \kappa)
$$

with the parameter $L_{B}=e^{2} /\left(4 \pi \varepsilon_{0}\right)$ (where $e$ is the elementary charge and $\varepsilon_{0}$ the permitivity of vacuum). Setting $\lambda=0$ gives a purely repulsive soft sphere interaction, while setting $\lambda=1$ recovers the full Lennard-Jones interaction. If we take $\sigma=3 \AA$ and $\varepsilon / k_{B}=85 \mathrm{~K}$ (both values compatible with most atomic liquids [23]), then $L_{B} / k_{B} \sigma \approx 55740 \mathrm{~K}$, which shows that the Coulomb interaction is about 655 times larger compared to the soft repulsion. It is this large discrepancy which is at the origin of the charge ordering dominating the random position of the atoms due to pure hard core effects, as in a uncharged atomic liquid.

We consider now the unscreened Coulomb, with $\kappa=+\infty$. To appreciate the nature of charge ordering relative to the charge itself, we increase the valence $z$ 
from 0 to 1 and look at the correlation functions and structure factors.However, the pure ionic liquid with valence $z=1$ is a liquid only at very high temperatures. In order to make the comparison meaningful, in terms of dense disordered liquid, we scale both the valence and the temperature such that the ratio $z^{2} / T \approx 1 / T_{I}$ where $T_{I}=4000 \mathrm{~K}$, the temperature of the ionic liquid with valence $z=1$. This gives $T=1000 \mathrm{~K}$ for $z=0.5, T=160 \mathrm{~K}$ for $z=0.2$, and we take $T=100 \mathrm{~K}$ for $z=0.02$ (to avoid temperatures below freezing). The results are represented in Fig.2. We note that, for $z=0$ exactly, this is just a single component LJ liquid since A and B represent the same system. It is seen that, as the Coulomb interaction becomes more prominent through the increase of $z$, the long range part of the like and cross correlations, namely $g_{++}(r)=g_{--}(r)$ and $g_{+-}(r)$, respectively, progressively move from perfectly in phase to exactly out of phase. Defining $h_{a b}(r)=g_{a b}(r)-1$, the fact that the long range part of the correlations are out of phase translates simply by the condition

$$
h_{++}(r)=h_{--}(r)=-h_{+-}(r) \quad r>1.2 \sigma
$$

for large distances. This illustrates charge order for Coulomb systems.

In order to further appreciate charge order, we examine the partial structure factors in the inset of Fig.1. The perfect alternation of out-of-phase correlations is reflected in the positive and negative peaks of the partial structure factors $S_{+-}(k)$ and $S_{++}(k)=S_{--}(k)$, defined through the Fourier transform of the $h_{a b}(r)$ :

$$
S_{a b}(k)=\delta_{a b}+\rho \sqrt{x_{a} x_{b}} \int d \vec{r} h_{a b}(r) \exp (\overrightarrow{i r} . \vec{k})
$$

where $\rho=N / V$ is the number density defined as the number $N$ or particle per volume $V$, and for the present model the mole fractions of the charges are $x_{a}=x_{b}=$ 0.5 . In the inset of Fig.1, the cross structure factor is intentionally shifted by 1 , in order to facilitate the comparison of the features complementary to the like structure factors $S_{a a}(k)$. We will keep this convention throughout the paper. The insets show two important features. The first feature, which interests us mostly in this paper, is the way the main peak at $k \sigma \approx 2 \pi$ at low valence $z=0.02$, changes into opposing sign peaks with increasing valence $z$-positive for the cross correlations and negative for the like correlations, and at the same time shift their position to an inner peak at $k \sigma \approx 5$. The opposing signs are simply the expression of Eq.(2) through the definition in Eq.(3). The Bhatia-Thornton representation, to be discussed in the next sub-section and illustrated in Fig.4, shows how these opposing contributions vanish exactly when summed, leaving the LJ main peak at $k \sigma \approx 2 \pi$ standing out. This main peak indeed disappears from the cross-correlations, since there are no longer like-charge correlations at contact $r=\sigma$. The second feature is the $k=0$ behaviour, which reflects the so-called Stillinger-Lovett sum 
rules[30, 31]. These rules come from the mathematical nature of the $1 / r$ form of the Coulomb interactions. It is notable that this form appears as soon as $z \neq 0$, for which both the cross and like structure factors differ the most, as seen for the case $z=0.02$.

How does charge ordering appears visually? Fig.2 shows a snapshot of the system at $z=0$ and at $z=1$. It is clearly seen that, while the first system has a random distribution of the spheres $\mathrm{A}$ and $\mathrm{B}$, the fully charge ordered system has a near perfect alternation of $\mathrm{A}$ and $\mathrm{B}$, yet being a disordered liquid. We are not sure that this remarkable form of order-within-disorder has been previously appreciated[3, 4]. Yet, this order is the key to explain several form of molecular ordering in liquids, as we will illustrate below. We wish to emphasize that this system has an almost ideal homogeneity and hence should display very little concentration fluctuations. We note in passing that, in the low density region, this system displays a liquid-vapour phase transition at low temperature. Indeed, at low densities, the charge attraction leads to a extremely inhomogeneous and clustered particle configuration, and charge order is visible only in the dense clusters. This is the reason we focus in the present work in the dense liquid phase, away from the fluid region of medium to low densities.

Next, we illustrate in Fig. 3 the relative independence of charge order from the long range part of the Coulomb interaction by considering screened Coulomb interactions, with various values of $\kappa$ in Eq.(1). The screening parameter $\kappa$ in Eq.(1) allows to control both the magnitude and the range of the Coulomb interaction through the screening factor $\exp (-r / \kappa)$. For $\kappa=+\infty$ we have the unscreened Coulomb. We note that for $\kappa / \sigma=1$ (left panel) the charge order is still intact, as seen by the out-of-phase correlations. It is only for $\kappa / \sigma<1$ that charge order weakens at long distances. We note that the LJ peak at $k \sigma \approx 2 \pi$ becomes more apparent as charge order is weakened (middle and right panels). We note that the $k=0$ part of the structure factors look very much as if they still obeyed the Stillinger-Lovett sum rules, while the $1 / \mathrm{r}$ mathematical singularity has disappeared. This appearance indicates that the very small-k behaviour of the structure factor is still dictated by the large magnitude discrepancy between the Coulomb and LJ interactions, rather by the mathematical singularity of the former interaction. This particular point explains why the short range interactions models of Nezbeda and co-workers[32, 33] for water and other associated liquids shared many of the characteristics of the corresponding point charge models.

\subsection{On the Bathia-Thornton - Pings-Waser formal equivalence}

In a charged binary system, only the global density is an observable because of the global electroneutrality. Indeed, it is not possible to add an excess of charged particles of one valence without breaking the equilibrium of the mixture. Such 
total instantaneous density is defined microscopically as $\rho_{N}(\vec{r})=\rho_{+}(\vec{r})+\rho_{-}(\vec{r})$. It is possible to define another density variable with witnesses the local charge $\rho_{Z}(\vec{r})=z_{+} \rho_{+}(\vec{r})+z_{-} \rho_{-}(\vec{r})$. Due to local fluctuations, this latter density is not necessarily zero at any given point of the mixture. These new random variables provide the basis for the Bathia-Thornton approach $[22,34]$ to generate new structure factors from the 3 correlation functions $g_{N N}(r), g_{Z Z}(r)$ and $g_{N Z}(r)$. The same way partial structure factors are defined by $S_{i j}(k)=<\rho_{i}(\vec{k}) \rho_{j}(-\vec{k})>$, one can build new structure factors by correlating these new density variables, which can be conveniently related to the atom-atom structure factors as[23, 34]:

$$
\begin{gathered}
S_{N N}(k)=\frac{1}{2}\left[S_{++}(k)+S_{--}(k)+2 S_{+-}(k)\right] \\
S_{Z Z}(k)=\frac{1}{2}\left[z_{+}^{2} S_{++}(k)+z_{-}^{2} S_{--}(k)+2 z_{+} z_{-} S_{+-}(k)\right]
\end{gathered}
$$

In terms of radiation scattering experiments, only the correlations related to the density-density structure factor are a macroscopic observable. This is true for Xray scattering experiments. Neutron scattering experiments allow to deuterate certain atoms and bias the resulting measured total structure factor, hence allowing to extract partial structure factors $[35,36]$.

It is interesting to observe that the Pings Waser (PW) form[37] of the total structure factor measured by radiation scattering, applied to the present case, is represented by

$$
I(k)=\sum_{i} x_{i} f_{i}(k)^{2}+\rho \sum_{i, j} x_{i} x_{j} f_{i}(k) f_{j}(k) \tilde{h}_{i j}(k)
$$

where the sum runs over all type of atoms $i$ and $j$ and the f-functions are the atomic form factors [38]. This expression is very similar to the density-density structure factor in Eq.(4), aside for the weights introduced by the form factors. This can be seen by rewriting the above expression as

$$
I(k)=\sum_{i j} \sqrt{x_{i} x_{j}} S_{i j}(k)
$$

with the structure factor $S_{i j}(k)$ defined as in Eq.(3). This form looks very much like Eq(4), enforcing a possible relation between the PW and BT expressions. The first term in Eq.(6) represents the ideal scattering $I_{\text {ideal }}(k)=\sum_{i} x_{i} f_{i}(k)^{2}$, which corresponds to neglecting contributions from correlations, or equivalently setting $\rho=0$.

We propose here that the various radiation scattering experiments measure an equivalent of the total density-density correlations, weighted by the form factors, which themselves reflect the specificity of the probing radiation. In other words, the total atomic density would be the microscopic random variable 


$$
\rho_{N}(\vec{r})=\sum_{i=1}^{N_{a}} \rho_{i}(\vec{r})
$$

where the index i runs over all the atoms in the liquid, independently of the underlying molecular structures, and where $N_{a}$ is the total number of atoms. This random variable can be weighted by convoluting appropriate form factors $f_{R}(\vec{r})$ depending on the specific form of radiation $R$ (Xray, neutron, light), creating a new total density:

$$
\rho_{R}(\vec{r})=\sum_{i=1}^{N_{a}} \rho_{i}(\vec{r}) \otimes f(\vec{r})=\sum_{i=1}^{N_{a}} \int d \vec{s} \rho_{i}(\vec{s}) f_{R}(\vec{r}-\vec{s})
$$

The Fourier transform of the convolution product leads to a multiplicative form seen in the PW expression. From these densities one could form appropriate structure factors

$$
S_{R R}(k)=<\rho_{R}(\vec{k}) \rho_{R}(-\vec{k})>
$$

following the usual procedure, where $<.>$ represents an ensemble statistical average[20]. Since the form factors do not enter the ensemble averaging, it is easy to see that this structure factor is exactly related to the PW scattering intensity

$$
I(k)=S_{R R}(k)
$$

In other words, the PW expression represents a radiation weighted equivalent of the BT structure factor, hence providing a generalisation of this quantity. Similarly, one could build a generalised PW expression for the charge-charge structure factor $S_{Z Z(k)}$, where the form factors $f_{i}(k)$ would be replaced by the atomic valence $z_{i}$. Conversely, it is not possible to build a generalisation of the cross structure factors, the main reason being that such quantities are not experimental observables. It could be possible to define generalisations which could be measured in computer experiment, but the purpose of such quantities is speculative for now.

We now build the BT-PW structure factors for the model of charge order studied previously. The left panel of Fig.4 shows both the density-density and chargecharge structure factors, as a function of charge ordering through the increase of valence $z$. It is seen that the $S_{N N}(k)$ looks exactly like a standard hard spheres structure factor, with a main peak about $k=2 \pi / \sigma$. The charge order peculiarities have disappeared by cancellation of the positive and negative peaks of the atomatoms structure factors displayed in Fig.1. It is the $S_{Z Z}(k)$ which has the charge order peak. The homogeneity of the charge order enforces its invisibility as an observable in $S_{N N}(k)$. The corresponding distribution functions in real space are 
shown in the right panel of Fig.4. Usually, these functions are not shown, because their definition and interpretation as correlation functions in the proper sense is not straightforward. For example, it is seen that the charge-charge $g_{Z Z}(r)$ is not zero inside the core and it has a negative first peak, which comes from the definition in $\mathrm{Eq}(5)$. Only the density-density function $g_{N N}(r)$ preserves the full meaning of a correlation function, because it is related to the microscopic observable $\rho_{N}(\vec{r})$. Regardless of these problems, these functions are interesting to study. For example, one sees that the packing correlations in $g_{N N}(r)$ vanish beyond $r / \sigma=1.5$ when charge ordering becomes important, as soon as $z \geq 0.5$. This is important to understand the absence of long range packing correlations in neat water[39], as we discuss in Section 4.2.

The main message of this section is to put forward that the homogeneity of the liquid enforced by charge order can be associated to a form of symmetry, a physical one, instead of a mathematical one. We now examine how this principle apply to more realistic dense liquids, and particularly if this new form of symmetry can be broken, and what would be the consequences.

\section{Charge order in ionic liquids and alcohols}

The purpose of this section is twofold. First, we show that the pre-peak in the partial charge-charge structure factors arise from a breakdown of the local homogeneity of charge order, due to molecular topology. Second, we show that these pre-peaks in the partial charge-charge structure factors contribute to the universal pre-peak seen the radiation scattering experiments, and this contribution is constructive instead of destructive as in the BT representation of charge order. This latter point has an heuristic importance, which we will discuss in the next section.

In this section, we use Molecular Dynamics simulation techniques, namely with the Gromacs package[40]. We use constant volume and temperature techniques, with a fixed number of particle of $\mathrm{N}=2048$ molecules. The initial configurations are generated by the PACKMOL program[41]. These configurations are equilibrated for $1 \mathrm{~ns}$, and subsequent production runs of $2 \mathrm{~ns}$ are used to extract accurate and noiseless correlation functions.

\subsection{Room temperature Ionic liquids}

In a previous work[23], we have addressed in some details the charge domain ordering in simply disordered ionic melts and complex disordered room temperature ionic liquids (RTIL). Simple ionic disorder is synonymous of the homogeneous charge order discussed above. Conversely, complex ionic disorder is characterised by a scattering pre-peak $[6,8,11,23]$, which is present in room temperature ionic 
liquids and absent from the high temperature ionic melts[3, 4]. This pre-peak has been associated to the segregated domains, as observed in early computer simulations of these systems[42], as well in models[43, 44]. In Ref.[23], we argued that it was the confinement of the charge order into small domains that was at the origin of a domain pre-peak in the various partial structure factors. This argument is in line with the general understanding of the scattering pre-peak observed in RTIL in terms of the polar/apolar segregation[45]. However, this argument is not completely correct, since the principal cause is argued here to come from a more general principle, which is symmetry breaking of the global homogeneity of charge order. This disruption of global charge order manifests itself by charge confinement into smaller domains, which appears to be a consequence of the symmetry breaking. The disruption of the homogeneity of charge order is due to the presence of neutral atomic groups attached to the anions in RTIL systems[6, 8, 23, 45].

We follow the coarse graining methodology of Voth and co-authors[10, 11] to introduce model RTIL molecules, similar to those we have previously adopted in Ref.[[23]]. We consider the same model as in the previous section, but we attach jointive soft spheres in a linear fashion to one side of the anion, making rod-like sphere chains, with one end charged. The intersphere distance is $l / \sigma=2.5 / 3$. The snapshot in Fig. 5 shows that the presence of neutral chains disrupt the homogeneous distribution of charges and produces their confinement in smaller volumes. A closer look at these configurations shows that the charged atoms prefer to dispose themselves in charge-alternated chains-like patterns. This is more true for longer anion chain molecules. This particular disposition has an impact on the form of the site-site distribution functions and structure factors, displayed in the left panel of Fig.5. It is clearly seen that the second and third neighbour correlations of the cross-correlations are depressed, due to the decrease of neighbours due to charge alignment. It is this depression of correlations which produces the pre-peak in the corresponding partial structure factors shown in the inset. The charge ordering peak at $k \sigma \approx 5$ is shown by the blue array, and is very similar to that seen in the simple ionic system of the previous sub-section. The pre-peak at $k \sigma \approx 1.3$ is shown by the red-array. It corresponds to spatial domains of extent $8 \sigma$, which correspond roughly to the visual domains seen in the snapshot. The LJ main peak is still seen only on the cross correlation structure factor, just like the simple ionic liquids, and for the same reasons. the most important feature of the domain pre-peaks is that they both have the same positive sign. In other words, they would both add up in the BT-PW representations.

This is shown in Fig.6, where we represent in the main panel different forms of the BT-PW intensities (with the ideal contributions Eq.(12) shown as dashed lines). The position of the pre-peak for each set of curves are signalled by a vertical line. The inset shows all the partial structure factors (with the same convention of shifting by 1 the cross correlations). In the main panel, the purple curve cor- 
responds to the BT form, where only the charged atoms contributions are added up. It shows the pre-peak at $k \sigma \approx 1.3$, this position signaled by vertical thin pink line, which is consistent with the corresponding structure factor features in the inset (thin vertical line at $k \sigma \approx 1.3$ ). The flat jade-green curve represents the ideal scattering in Eq.(6). The cyan curve represents the same BT form, but with all the structure factors represented in with the same weight of 1 . In this representation, the domain pre-peak become a weak shoulder at $k \sigma \approx 2.7$, while the LJ peaks at $k \sigma \approx 5-6$ acquire the most importance. This is simply because the neutral carbon group contributions become predominant, and they have nearly canceling contributions when added to the cross correlations with charged groups (see inset). The blue curve represents the XRay-like scattering representation, with the appropriate form factors, with the oxygen representation of the anion and the anion, and carbon for the neutral atoms. The dashed green line is the ideal contribution, which has a non-trivial k-dependence because of the form factors $f_{i}(k)$, as opposed to the previous cases discussed above where this curve was flat. In this representation the shifted pre-peak contributes predominantly at $k \sigma \approx 2.7$ (vertical thick red line), while the LJ contributions at $k \sigma \approx 5-6$ show as a weak shoulder(vertical thin pink line). The principal contribution at $k \sigma \approx 1.3$ (see inset), quite apparent in the BT scattering representation, has been blurred by contributions of the form factors of the carbon groups, which enhance the contribution at $k \sigma \approx 2.7$. It is note worthy that the decaying of the form factor at large $\mathrm{k}$ affect the shape of this scattering function more realistically than the model BT-like intensities. It is noteworthy that the BT pre-peaks in the atom-atom structure factors in the inset (marked by the green vertical line) cancels in the total representation.

\subsection{Alcohols}

We consider 3 models of alcohols (Fig.7), starting with the sole hydroxyl group (model 1), and build from it methanol and ethanol like models by attaching 1 and 2 more soft neutral spheres, at an angle of 109 degree from the hydroxyl charges axis. Fig. 7 shows a picture of these toy alcohol models. Model 2 would be akin to methanol while model 3 to ethanol. The overall interaction between different units is the same as in Eq.(1), with appropriate charge parameters for the various groups, and setting $\lambda=1$ in order to have a Lennard-Jonesium, just like in classical models of alcohol molecules. In Model 1, the toy-hydrogen site is modeled as a pure charge, with valence $z_{H}=+0.45$, while the toy-oxygen group is a LennardJonesium, with the same $\varepsilon / k_{B}$ and $\sigma$ parameters as in the previous models, with valence $z_{O}=-0.45$.This way, the model hydroxyl group is charge neutral, unlike most alcohol models, such as the Optimized Potentials for Liquid Simulations (OPLS)[46] and Transferable Potential for Phase Equilibria (TraPPE)[47]. This is however enough the capture the structural features of realistic alcohols, as 
demonstrated below. We choose molar volumes for dense liquids as $V_{m 1}=18.75$ $\mathrm{cm}^{3} / \mathrm{mol}, V_{m 2}=32.4 \mathrm{~cm}^{3} / \mathrm{mol}$ and $V_{m 1}=57.16 \mathrm{~cm}^{3} / \mathrm{mol}$, for model 1 , and 3, respectively. This leads to box sizes of $L_{1}=40 \AA, L_{2}=48 \AA L_{3}=52 \AA$, respectively, for a number of particles of $N=2048$.

Fig.7 equally shows snapshots of the three models, highlighting the hydroxyl groups with a white sphere for the hydrogen and a blue sphere for the oxygen sites, while the neutral groups are shown as semi-transparent cyan spheres. It is seen that, in all the snapshots, the hydroxyl groups tend to form chains, as would be expected from a fluid of pure dipolar sites[48, 49, 50, 51]. However, these chains become less pronounced when the neutral group tail is increased. Conversely, in the case of model 1 , one sees that the rather short chains tend to align anti-parallel to each other, which is a required constraint in a dense chain liquid of dipoles, since dipole prefer to be anti-parallel next to each other. This constraint is relieved when neutral atoms are added, leading to lone chains, which are longer. This is an important difference in terms of charge ordering. Indeed, despite the chain formation in the pure hydroxyl group liquid, the charge ordering is globally distributed. When neutral groups are present, local isotropy of charge order becomes broken, and lone chains appear. The more numerous the neutral background particles, the better isolated chains. In these chains, charge order exist but has lost spatial isotropy, hence the depletion of second and third neighbour correlations. It is this feature which is at principal origin of the pre-peaks, as seen in Fig.8.

Fig. 8 shows the correlation functions between the charged groups for the 3 models, as well as the corresponding structure factors in the insets. The correlations between the - sites are drawn in blue, those between the + site are in magenta, and the +- cross correlations shown in dashed green lines. The different panels show that the correlation functions develop features similar to that of the ionic liquids examined in the previous section 3 . The first peak of the cross correlation $g_{+-}(r)$ increases with charge confinement in chains, while the second and third peaks are depressed. This feature expresses that the +- correlations increase due to higher localization in smaller pockets, as seen in Fig.7 central and right snapshots. The case of the pure hydroxyl alcohol (model 1) shows only first peak increase. The structure factors (shown in the insets) also reflect the localised charge ordering, just like ionic liquids. In the case of model 1 (left panel) we see the BT-like feature centered at $k_{B T} \sigma \approx 8$, which corresponds to the distance between to oppositely oriented dipoles. This is shown by the yellow vertical line. The main peak is at $k_{M} \sigma \approx 2 \pi$, as in a usual LJ system. The most interesting result if is the BT structure factor, built from only the charged hydrogen and oxygen like sites, as in Eq.(4). This is shown in darker vertical line. One sees clearly that the main peak is centered at $k_{M} \sigma \approx 2 \pi$, while the BT peak has totally disappeared, by cancellation of the,++-- and +- contributions. The situation is 
radically different in the insets of the middle and right panels, where chains are isolated from neutral groups. The BT peaks are again seen very clearly again at $k_{B T} \sigma \approx 8$, by the out of phase positive peaks(for +- ) and negative peaks for $(++$ and --). But now, we have a pre-peak, which depends of the system (shown in thicker vertical lines). These pre-peaks appear positively in each partial structure factors, and not destructively as for the BT peaks. In that, they witness features common to all correlations, namely the existence of confined charges. Interestingly, these pre-peak equally appear in the correlations between the neutral sites (more pronounced for the sites closer to the charged ones than those at the tail), witnessing the fact that neutral site domains complement those of charged sites. The position $k_{P}$ of the pre-peak indicates the average domain size $d$ through the Fourier relation $k_{P} d \approx 2 \pi$, which is why it is system dependent. The main LJ peak is not very apparent, as compared to methanol[52] and ethanol[53], but this is probably due to the model design. Merging the neutral sites help get more pronounced main peaks. Indeed, long chains are known to have very weak main peak in their structure factors[54].

Finally, Fig.9 shows the toy SAXS scattering data for all 3 models. These are computed by attributing the from factor of hydrogen to the positive hydrogenlike site, and oxygen for the negatively charged oxygen-like site, and carbon form factor to all the neutral sites. Green lines are the ideal contributions in Eq.(6):

$$
I_{\text {ideal }}(k)=\sum_{i} f_{i}(k)
$$

where $x_{i}$ is the site mole fraction. The total $\mathrm{I}(\mathrm{k})$ are shown in blue lines. One sees that the scattering data for model 1 has a main peak at $k_{M} \sigma \approx 2 \pi$, which is $k_{M} \approx 2$ $\AA^{-1}$ for $\sigma=3 \AA$. The BT positive and negative peaks have totally disappeared, just like for the BT structure factor in Fig.8. The pre-peaks become apparent for the SAXS intensities of model 2 and model 3, which positions similar to those seen in the corresponding structure factors shown in Fig.8. It is seen that the prepeak of methanol-like model 1 is at larger wave vector $k \approx 1.25 \AA$ than that of the ethanol-like model 3 , at $k \approx 0.8 \AA$. This is compatible with the experimental observation that the pre-peak position of longer mono-ols shift to smaller wave vectors, indicating larger domains. The main peak positions vary around the ideal position $k_{M}$.

An intriguing feature is equally observed, with negative values at small-k vectors for model 2 and 3, which is in variance with experimental data. The enhanced negative contributions come from the dominating importance of the crosscorrelations, which have negative $k=0$ values, as usual. This gives some clues. In realistic systems, these contributions may be tuned such that the $\mathrm{k}=0$ intensity is always positive. For example, this could be a model artifact, and due to attributing the form factor of carbon to these sites. Decreasing the density reduces the excess 
contributions and make $\mathrm{I}(\mathrm{k}=0)>0$. However, despite many possible rationalisation to this behaviour, it remains the suspicion that the PW expression may be incorrect, because it does not incorporate the fact that molecular liquids are not free atom mixtures, and the fact that particular atoms form molecules should somehow reflect in the total scattered intensity, regardless of the way it is obtained. Indeed, if we account for intra-molecular contributions, these act as positive contributions at $k=0$, for structure factors $S_{a b}(k)$ between two distinct atoms $a$ and $b$ inside the same molecule, with the simplest form being the zeroth-order Bessel function $j_{0}\left(k d_{a b}\right)$, where $d_{a b}$ is the distance between the two atoms. We will examine this point in a subsequent work.

\section{Discussion}

We briefly examine in this section the mathematical origin of the pre-peak as well as the case of other types of associating liquids such as water and core-soft models, but under the angle of charge-ordering.

\subsection{Mathematical origin of the pre-peak}

Until now, we have give a rational physical explanation to the arousal of a prepeak, due to confinement of charge ordering. In order to understand the mathematical origin of the pre-peak, we consider the RDF of a dense soft sphere fluid (for reduced density and temperatures $\rho^{*}=0.8$ and $T^{*}=0.9$ ), together with its associated structure factor, as shown in magenta curves in Fig.10. We now add to the RDF function a narrow high positive Gaussian $A_{1} \exp \left(-\left(r-r_{1}\right)^{2} / \kappa_{1}^{2}\right)$ centered at $r_{1}=1.1 \sigma$, and a broad smaller negative Gaussian $-A_{2} \exp \left(-\left(r-r_{2}\right)^{2} / \kappa_{2}^{2}\right)$ centered at $r_{2}=2 \sigma$. We consider the parameter pairs $A_{1}=2.5, \kappa_{1}=96 A_{2}=0.15$, $\kappa_{1}=3$. These functions and their Fourier transforms are shown in orange. The purpose of the first Gaussian is to mimic the increase of the first peak due to specific directional attraction, while the second Gaussian will decrease the second and third neighbour correlations, mimicking the depletion mechanism mentioned in the previous sections. This is equally shown in blue lines in Fig.10. It is seen that the Fourier transforms of these 2 opposite sign Gaussian compete to produce a net positive peak between $k=0$ and $k_{M}=2 \pi / \sigma$, as indicated by the red arrow, and demonstrating the origin of the pre-peak. The blue arrow shows that the main peak remains unaffected by these transformation. Since this is only an approximate approach, it is expected that it will not produce a physical structure factor, hence the negative parts of the resulting structure factor. However, it should help capture the pre-peak feature through the physically relevant Gaussian introduced to capture the geometrical constraints on the charge order. 
The 3D Fourier transform of shifted Gaussian is clearly not a Gaussian function. However, assuming that they are approximately Gaussian at about the prepeak position, one can relate the position of the pre-peak to the magnitude $A_{i}$ and width $\kappa_{i}$ parameters of the two Gaussian by:

$$
k_{P} \approx \sqrt{\frac{1}{\kappa_{2}^{2}-\kappa_{1}^{2}} \ln \left(\frac{A_{2} \kappa_{2}^{2}}{A_{1} \kappa_{1}^{2}}\right)}
$$

This expression shows that the pre-peak position is at least controlled by the $\kappa_{2}^{-1}$, hence the width of the depression of the second and higher neighbours.

\subsection{Water and aqueous mixtures}

The tetrahedrality of water charge distribution ensures a good isotropy of the charge ordering. Hence, we do not expect to see any pre-peak. Indeed, the oxygen-oxygen structure factor of water shows a split main peak, the outer one associated to the contact distance $\sigma \approx 3 \AA$ and the inner-one to the Hbonding distance $d=2 \AA$ [39]. It would be interesting to re-examine the properties of water in terms of charge ordering, instead of the directional hydrogen bonding and associated network pictures, which have been previously used [55, 56, 57, 58, 59, 60]. The charge order picture is connected with shapes of clusters, such as chains, instead of networks, particles, instead of bonds. One point that was raised in the previous work[39] was the absence of packing correlation in $g_{W W}(r)$ beyond $r \approx 9 \AA$, which is intriguing in a densely packed liquid such as water. The data shown in Fig.4 right panel provides an explanation. It shows that correlation of the total density can vanish at large distance because they encompass partial charge correlations which are in phase opposition. It is the fact that, for classical water model, the charges are buried inside the single LJ sphere, and yet enough far apart to contribute to charge oscillations in the short range correlations, that we observe this peculiarity of correlations in water.

The case of aqueous mixtures is more delicate. From our previous studies, we have seen that most aqueous mixtures show a strong domain pre-peak in the oxygen-oxygen structure factor of water. From the previous arguments, we expect that solute would interfere the isotropy of the charge ordering of water. The whole problem is how. This depends on the particular type of solutes. What we observe is that solute molecules such as alcohols make water to form compact segregated domains. We will address such system in a separate paper since the mechanism of charge ordering is very different than that exposed here. However, in some particular cases, such as with DMSO and amines, water forms chainlike aggregates[61, 62]. In such case, we observe the same feature described here, namely an increase of the first peak in oxygen-oxygen correlations and a depletion 
of correlations in the second neighbours. This explain the origin of the structure factor pre-peak that we have reported in previous works[61, 62].

\subsection{Core-Soft models}

Soft core models propose to mimic the local order, produced by hydrogen bond based molecular association, through two cores with soft and hard repulsion, both in $3 \mathrm{D}[59,60]$ and $2 \mathrm{D}[63,64]$. The outer soft core allows to break the isotropy and homogeneity of particle around the central particle, in order to optimise global repulsive contributions. This leads to effective clustering induced par pure repulsion[63, 64]. This picture is closer to the charge order picture, except that it is achieved with one type of particle instead of two, with opposite charges. But the principal idea is preserved, that of breaking isotropy of particle distribution. The fact that both methods produce pre-peak in relation to the clusters that appear, illustrates the link between symmetry and clustering. Indeed, in a recent work[65], we have shown that the core-soft repulsion can be extracted as an effective 1-component interaction from a binary mixture of site-site interaction model with preferential attraction, and which has a cluster pre-peak. However, soft-core models have limitations when it comes to mixtures such as aqueous mixtures or mixtures of associating species with neutral ones[66].

\section{Conclusion}

The physical phenomena of hydrogen bonding association, present in liquids such as water and alcohols, for example, has a simple underlying picture, which bring such liquids in par with ionic liquids, and this picture is that of charge ordering. The underlying idea is that plus and minus charges prefer to sit next to each other, which leads to a very particular ordering of the molecules, even in a disordered, isotropic and homogeneous liquid. This ordering does not require the long range nature of the Coulomb interaction, it only require that the interactions have a large magnitude near contact, larger that the Lennard-Jones repulsion and attractions. Attaching charges into rigidly bound positions in molecules, creates richer forms of charge order. In addition the presence of neutral groups induce frustration, and richer patterns of ordering, such a chain-like charge ordering. Here, we have shown that chain-like ordering is the dominant form of charge ordering in alcohols and room temperature ionic liquids. This special form of ordering affect the form of the correlation functions beyond first neighbours and is at the origin of the prepeak observed in both the individual atom-atoms structure factors (especially the charged atoms) and also the resulting scattering intensity. With respect to this measurable observable, we have shown that the Bhatia-Thorton structure factor 
bear appealing and unifying analogies with the scattering intensity, thus extending the BT formalism which is originally restricted to binary-mixtures.

One of the important heuristic questions concern the nature of charge order in relation to density or concentration fluctuations. The formal answer would be that the latter types of fluctuations are well defined within statistical thermodynamics and concern only the $\mathrm{k}=0$ part of the structure factor and scattered intensity. In such context, non-zero parts of the structure factors are related to wave-vector dependent fluctuations, which in turn correspond to fluctuations associated to particular inter-molecular distances. The appearance of a pre-peak, hence witness the emergence of a characteristic association length. It is important to understand that this length has nothing to do with the correlation length, which is by definition associated to the small wave vector behaviour of the structure factor near $\mathrm{k}=0$ [67]. This latter length concerns the scale at which macroscopic thermodynamic fluctuations are correlated, while the distance associated to the pre-peak concerns the specific forms of correlations between atoms, such as chains, for example. It is obvious that these two lengths are related through the microscopic processes specific to the types of liquids investigated. Finding their interrelation is an interesting topic for future investigations.

Finally, we note that the type of clustering we have discussed here concern the segregation between atomic groups belonging to the same molecule. The extension of this segregation to that observed in the case of aqueous mixtures, where two different type of molecules segregate from one another, while keeping global miscibility, -the so-called micro-heterogeneity- is not as direct as what we have addressed here. In particular, while the present work reveals the origin of the prepeak observed in real scattering experiments, in relation with the pre-peaks that appear in atom-atom structure factors, it remain to explain, why such pre-peaks are never seen in scattering data for simple aqueous mixtures, while atom-atom structure factors calculated from computer experiments appear to show the existence of pre-peaks associated to domain segregation[68].

\section{References}

[1] Edwards F G, Enderby J E and Page D I 1975 J . Phys. C: Solid State Phys. 83483

[2] N. H. March and M. P. Tosi, Atomic Dynamics in Liquids (Macmillan, London, 1976)

[3] M. Baus and J.-P. Hansen, Physics Reports 59, 1 (1980)

[4] J. E. Enderby and G. W. Neilson, Rep. Prog. Phys., 44, 38 (1981) 
[5] A. Triolo, O. Russina, H-J Bleif and E. Di Cola, J. Phys. Chem. B111, 4641 (2007)

[6] A. Triolo, O. Russina, B. Fazio, R. Triolo and E. Di Cola, Chem. Phys. Lett. 457, $362(2008)$

[7] H. V. R. Annapureddy, H. K. Kashyap, P. M. De Biase and C. J. Margulis, J. Phys. Chem. B114, 16838 (2010)

[8] C. S. Santos, H. V. R. Annapureddy, N. S. Murthy, H. K . Kashyap, E. W. Castner and C. J. Margulis, J. Chem. Phys. 134, 064501 (2011)

[9] H. K. Kashyap, C. S. Santos, H. V. R. Annapureddy, N. S. Murty, C. J. Margulis and E. W. Castner, Faraday Discuss. 154, 133 (2012)

[10] Y. Wang and G; A. Voth, J. Am. Chem. Soc. 12712192 (2005)

[11] Y. Wang, W. Jian, T. Yan and G. A. Voth, Acc. Chem. Res. 40, 1193 (2007)

[12] B. E. Warren, Phys. Rev. 44, 969 (1933).

[13] A. H. Narten and A. Habenschuss, J. Chem. Phys. 80, 3387 (1984)

[14] A. H. Narten and S. I. Sandler, J. Chem. Phys. 71, 2069 (1979)

[15] D. G. Montague, J. C. Dore and S. Cummings, Mol. Phys. 53, 1049 (1984)

[16] S. Sarkar and R. N. Joarder, J. Chem. Phys. 99, 2032 (1993)

[17] R. Böhmer, C. Gainaru and R. Richert, Phys. Rep. 545, 125 (2014)

[18] P. W. Anderson, Science 177, 393 (1972)

[19] P. M. Chaikin and T. C. Lubensky, Principles of condensed Matter Physics, Cambridge, Cambridge University Press 1995.

[20] J.P. Hansen and I.R. McDonald, Theory of Simple Liquids (Academic, London, 1986)

[21] M. Tomšič, A. Jamnik, G. Fritz-Popovski, O. Glatter and L. Vlcek, J. Phys.Chem. B111, 1738 (2007)

[22] A. B. Bhatia and E. E. Thornton, Phys. Rev. B2, 3004 (1970)

[23] A. Perera and R. Mazighi, J. Chem. Phys., 143, 154502 (2015)

[24] Y. Levin and M. E. Fisher, Physica A 225, 164 (1996) 
[25] J. M. Caillol, J. Chem. Phys. 100, 2161 (1994)

[26] G. Orkoulas and A. Z. Panagiotopoulos, J. Chem. Phys. 101, 1452 (1994); ibid J. Chem. Phys. 110, 1581 (1999)

[27] Q. Yan and J. de Pablo, J. Chem. Phys. 111, 9509 (1999)

[28] S. Wiegand, M.E. Briggs, J.M.H. Levelt Sengers, M. Kleemeier, W. Schröer, J. Chem. Phys. 1099038 (1998)

[29] W. Schröer, M. Wagner and O. Stanga, J. Mol.Liquids 1272 (2006)

[30] F. H. Stillinger and R. Lovett, J. Chem. Phys. 48, 3858 (1968)

[31] H. Van Beijeren and B. U. felderhof, Mol. Phys. 38, 1179 (1979)

[32] J. Jirsak and I. Nezbeda, J. Chem. Phys. 127, 124508 (2007)

[33] M. Kettler, I. Nezbeda, A. A. Chialvo and P. T. Cummings, J. Phys. Chem B 106, 7537 (2202)

[34] V. Bitrian, J. Trullas and M. Silbert, Physica B 403, 4249 (2008)

[35] A. K. Soper, Chem. Phys. 202, 295 (1996)

[36] T. Weitkamp, J. Neuefeind, H. E. Fisher and M. D. Zeidler, Mol. Phys. 98, 125 (2000)

[37] J. C. Pings and J. Waser, J. Chem. Phys. 48, 3016 (1968)

[38] International Tables for Crystallography, ed. E. Prince, International Union of Crystallography, 2006, vol. C.

[39] A. Perera, Mol. Phys. 109, 2433 (2011)

[40] D. van der Spoel., E. Lindahl, B. Hess., G. Groenhof, A. E. Mark and H. J. C. Berendsen, 2005, J.Comp. Chem. 26, 1701

[41] J. M. Martínez and L. Martínez, Journal of Computational Chemistry, 24(7):819-825, 2003; ibid L. Martínez, R. Andrade, E. G. Birgin, J. M. Martínez. Journal of Computational Chemistry, 30(13):2157-2164, 2009.

[42] J. N. Canongia Lopes, A. A. H. Pádua, J. Phys. Chem. B 1103330 (2006)

[43] G. A. Chapela, O. Guzmán, E. Díaz-Herrera, and F. del Río, J. Chem. Phys. 142, 154508 (2015) 
[44] O. Guzmán, J. Eloy Ramos Lara, and F. del Río J. Phys. Chem. B 119, 5864 (2015)

[45] O. Russina, A. Sferrazza, R. Caminiti and A. Triolo, J. Phys.Chem. Lett. 5, 1738 (2014)

[46] W. L. Jorgensen, J. Tirado-Rives, J. Am. Chem. Soc. 1657 (1988)

[47] B. Chen, J.J. Potoff and J.I. Siepmann, J. Phys. Chem. B 105, 3093-3104 (2001)

[48] D. Levesque and J. J. Weis, Phys. Rev E49, 5131 (1994)

[49] Y. Levin, Phys. Rev. Lett. 83, 1159 (1999)

[50] T. Tlusty and S. A. Safran, Science, 290, 1328 (2000)

[51] G. Ganzenmuller, G. N. Patey, P. J. Camp, Mol. Phys. 107, 403 (2009)

[52] L. Zoranic, F. Sokolic and A. Perera, J. Chem. Phys., 127, 024502 (2007).

[53] M. Mijaković , K. D. Polok, B. Kežić , F. Sokolić , A. Perera and L. Zoranić, Molecular Simulation 42 , 699 (2014)

[54] L. Laridjani and P. Lelouboucher, PLoS One 9, e109403 (2014)

[55] H. S. Frank and A. S. Quist, J. Chem. Phys. 34, 604 (1961)

[56] H. E. Stanley et al. J. Phys. Cond. Mat. 12, A403 (2000)

[57] H. E. Stanley, S. V. Buldyrev, M. Canpolat, O. Mishima, M. R. SadrLahijany, A. Scala and F. W. Starr, PCCP 2, 1551 (2000).

[58] P.G. Debenedetti Nature, 392, 127, (1998).

[59] E. A. Jagla, Phys. Rev. E 58, 1478 (1998).

[60] R. Lynden-Bell and P. G. Debenedetti, J. Phys. Chem. B 109, 6527 (2005)

[61] A. Perera and R. Mazighi J. Chem. Phys. 143, 154502 (2015)

[62] M. Pozar and A. Perera (accepted in J. Mol. Liq)

[63] G. Malescio and G. Pellicane, Nat. Mater. 2, 97 (2003).

[64] A. Perera, Mol. Phys. 107, 487 (2009). 
[65] C. Bores, E. Lomba, A. Perera, and N. Almarza, J. Chem. Phys. 143, 084501 (2015)

[66] B. Kežić, S. Dartois and A. Perera, Mol. Phys. 113, 1108 (2015)

[67] M. E. Fisher, J. Math. Phys. 5, 944 (1964)

[68] A. Perera, Pure Appl. Chem. 88, 189 (2016) 


\section{Figure captions}

Fig.1- Correlation functions (main panel) and structure factors (insets) of a binary mixture of charged soft spheres, for different valences $z \cdot g_{+-}(r)$ shown in magenta lines and $g_{++}(r)=g_{--}(r)$ shown in blue lines, respectively. The insets show the partial structure factors $S_{+-}(k)$ and $S_{++}(k)=S_{--}(k)$.

Fig.2- Snapshot of the binary mixture of charged soft spheres for $z=0$ and $z=1$. It illustrates the ordered nature of disordered liquid (see text)

Fig.3- Correlation functions (main panel) and structure factors (insets) of a binary mixture of screened charged soft spheres, for different values of the screening parameter $\kappa$, with same conventions as in Fig.1.

Fig.4- Bhatia-Thorton structure factors (left panel) and correlations (right panel) corresponding to the correlations and partial structure factors shown in Fig.1. Main panel for density-density N-N correlations and inset for charge-charge $\mathrm{Z}-\mathrm{Z}$ correlations. Blue line for $z=4$, orange for $z=0.5$, green for $z=0.2$ and cyan for $z=0.02$.

Fig.5- Left panel: model room-temperature ionic liquid and snapshots; right panel: correlation functions (main panel) and structure factors (inset). The arrows indicate the pre-peak (red) and main peak (blue) -see text.

Fig.6- Various types (see text for details) of scattering intensities for the model RTIL shown in Fig.5. For each data set, both the ideal(dashed line) and total(full line) contributions are shown. The inset shows all the site-site structure factors, with blue lines for the charged sites, black lines for the uncharged and orange for the charged-uncharged cross correlations. The vertical lines describes the various peaks discussed in the text.

Fig.7- Model alcohol molecules and corresponding snapshots of liquid states. The positively charged site is in white, the negative site in blue and the neutral sites in semi-transparent cyan.

Fig.8- Selected correlation functions (main panel) and structure factors (insets) of the 3 model alcohols shown in Fig.7. $g_{--}$shown in blue, $g_{++}$in magenta and $g_{+-}$in green dashes (same conventions for the structure factors). The vertical lines in the insets indicate the main and BT peaks (yellow) and prepeaks (orange) discussed in the text.

Fig.9- Xray scattering functions for the model alcohols shown in Fig.7. Green lines for the ideal contribution and blue lines for total intensities. Full lines 
for model 1, dotted for model 2 and dashed for model 3, as labelled next to the appropriate lines.

Fig.10- Illustration of the origin of the pre-peak (see text) through transformation of the correlation function in real space (main panel) and Fourier space (inset). The original correlations are shown in magenta, the transformed one in blue, and the added Gaussian functions and their transforms in orange. The blue arrow in the inset shows the position of main peak and the red arrow the position of the pre-peak. 
.Fig-1- Correlation functions (main panel) and structure factors (insets) of a binary mixture of charged soft spheres, for different valences $z \cdot g_{+-}(r)$ shown in magenta lines and $g_{++}(r)=g_{--}(r)$ shown in blue lines, respectively. The insets show the partial structure factors $S_{+-}(k)$ and $S_{++}(k)=S_{--}(k)$.
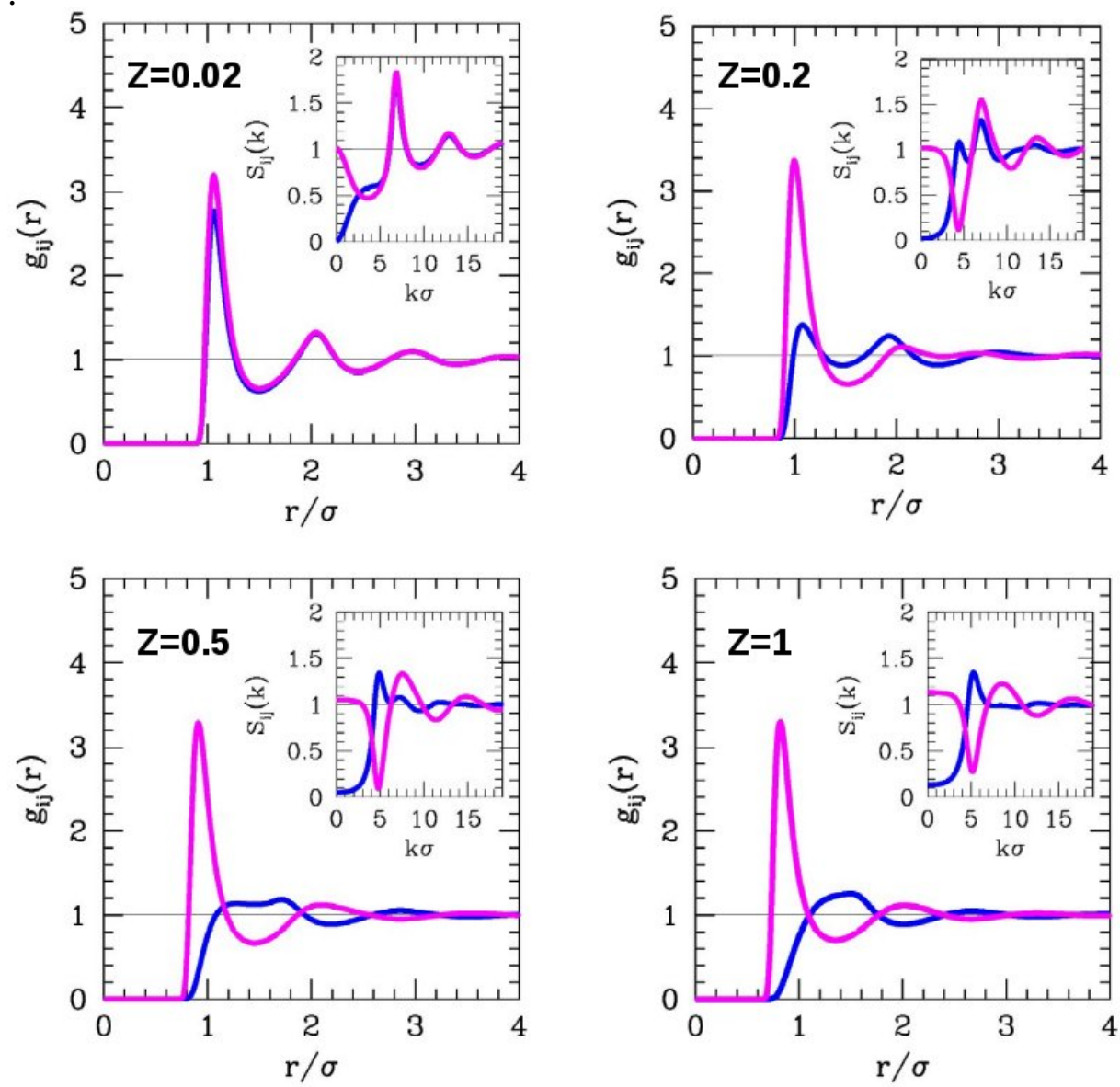
.Fig-2-Snapshot of the binary mixture of charged soft spheres for $z=0$ and $z=1$. It illustrates the ordered nature of disordered liquid (see text)

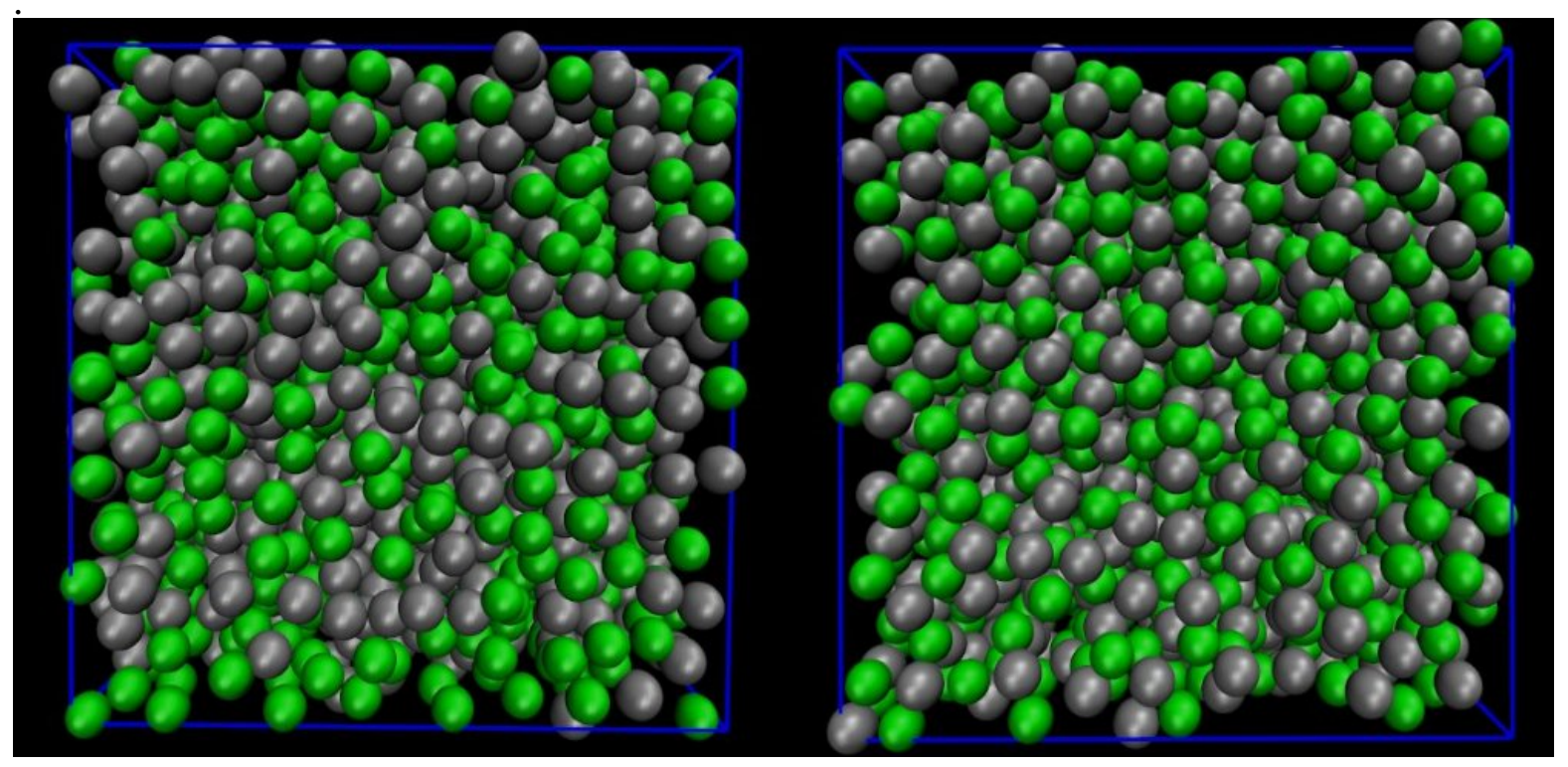


.Fig-3-Correlation functions (main panel) and structure factors (insets) of a binary mixture of screened charged soft spheres, for different values of the screening parameter $\kappa$, with same conventions as in Fig.1.

$$
K=1
$$

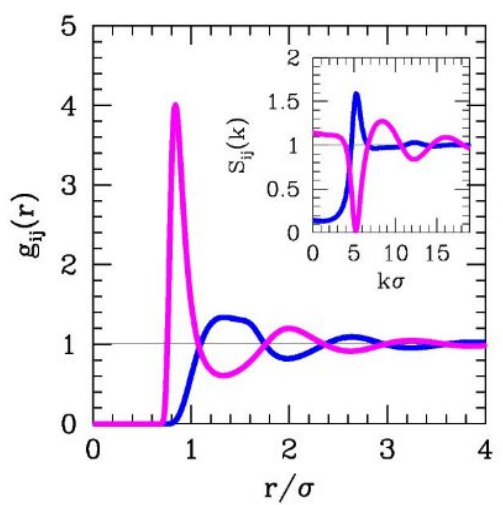

$K=1 / 3$

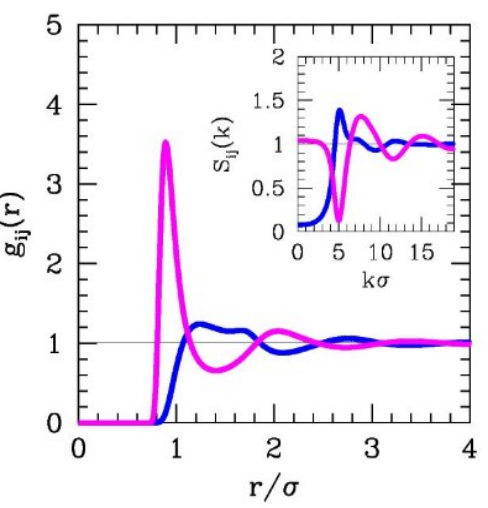

$\mathrm{K}=0.2$

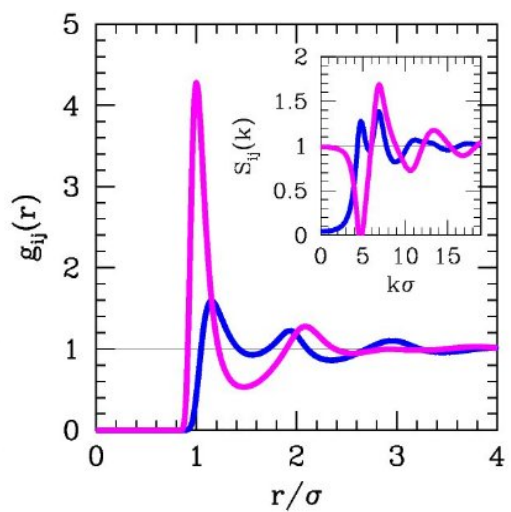


.Fig-4-Bhatia-Thorton structure factors (left panel) and correlations (right panel) corresponding to the correlations and partial structure factors shown in Fig.1. Main panel for density-density N-N correlations and inset for charge-charge Z$\mathrm{Z}$ correlations. Blue line for $z=4$, orange for $z=0.5$, green for $z=0.2$ and cyan for $z=0.02$.
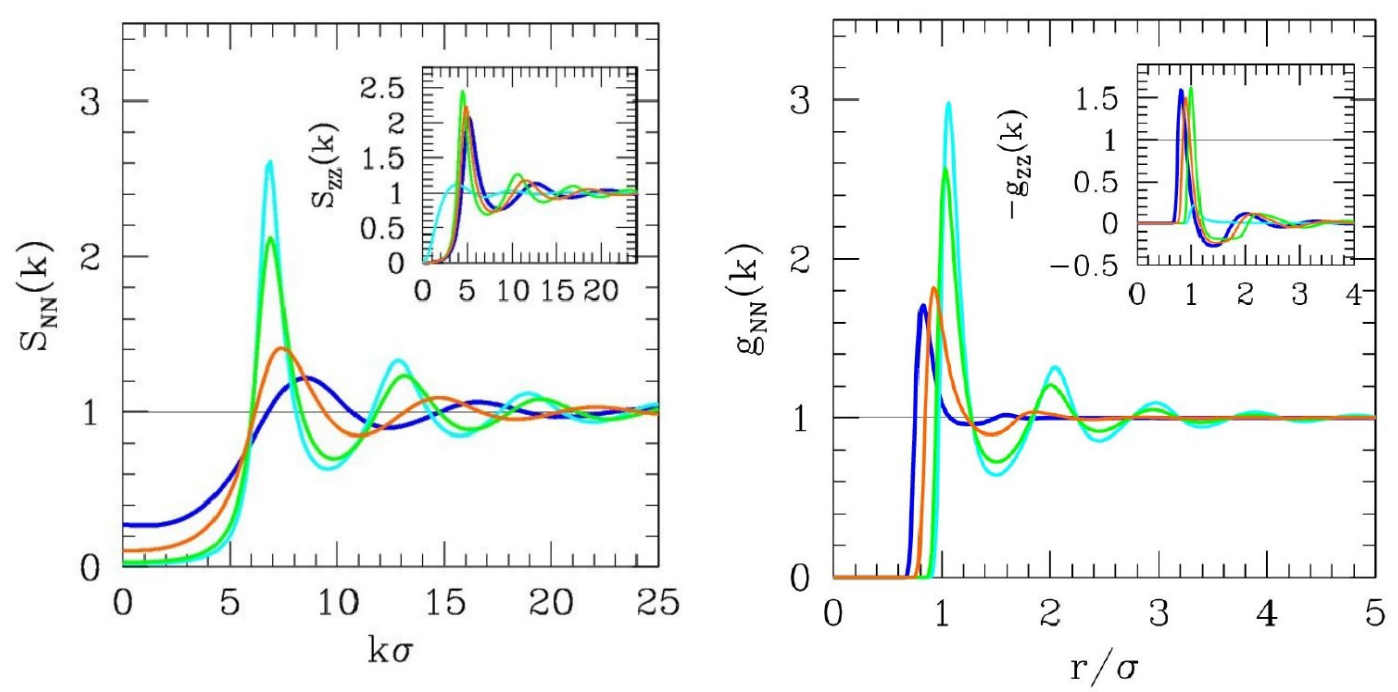
.Fig-5- Left panel: model room-temperature ionic liquid and snapshots; right panel: correlation functions (main panel) and structure factors (inset). The arrows indicate the pre-peak (red) and main peak (blue) -see text.
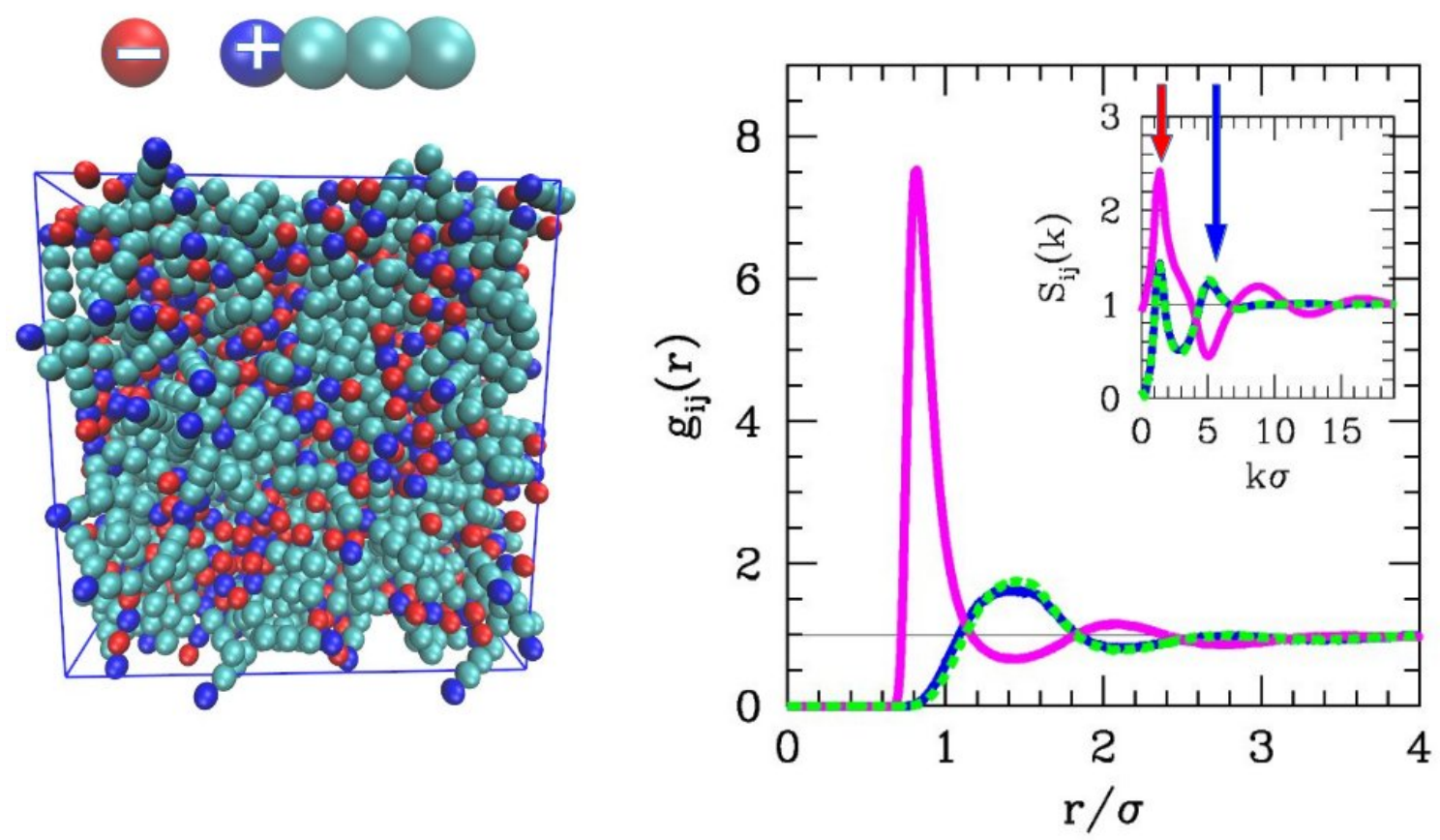
.Fig-6- Various types (see text for details) of scattering intensities for the model RTIL shown in Fig.5. For each data set, both the ideal(dashed line) and total(full line) contributions are shown. The inset shows all the site-site structure factors, with blue lines for the charged sites, black lines for the uncharged and orange for the charged-uncharged cross correlations. The vertical lines describes the various peaks discussed in the text. .

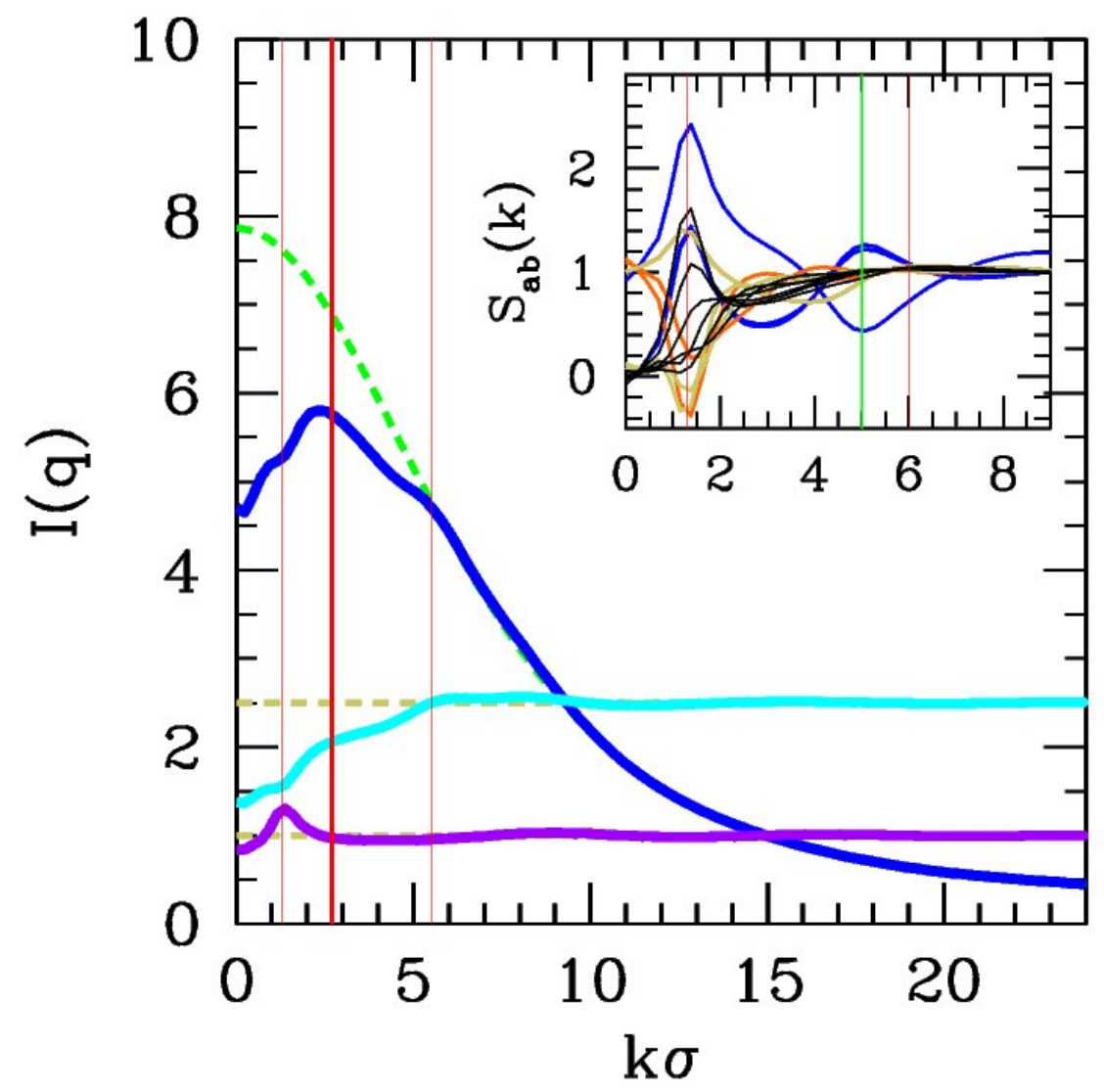


.Fig-7-Model alcohol molecules and corresponding snapshots of liquid states. The positively charged site is in white, the negative site in blue and the neutral sites in semi-transparent cyan.
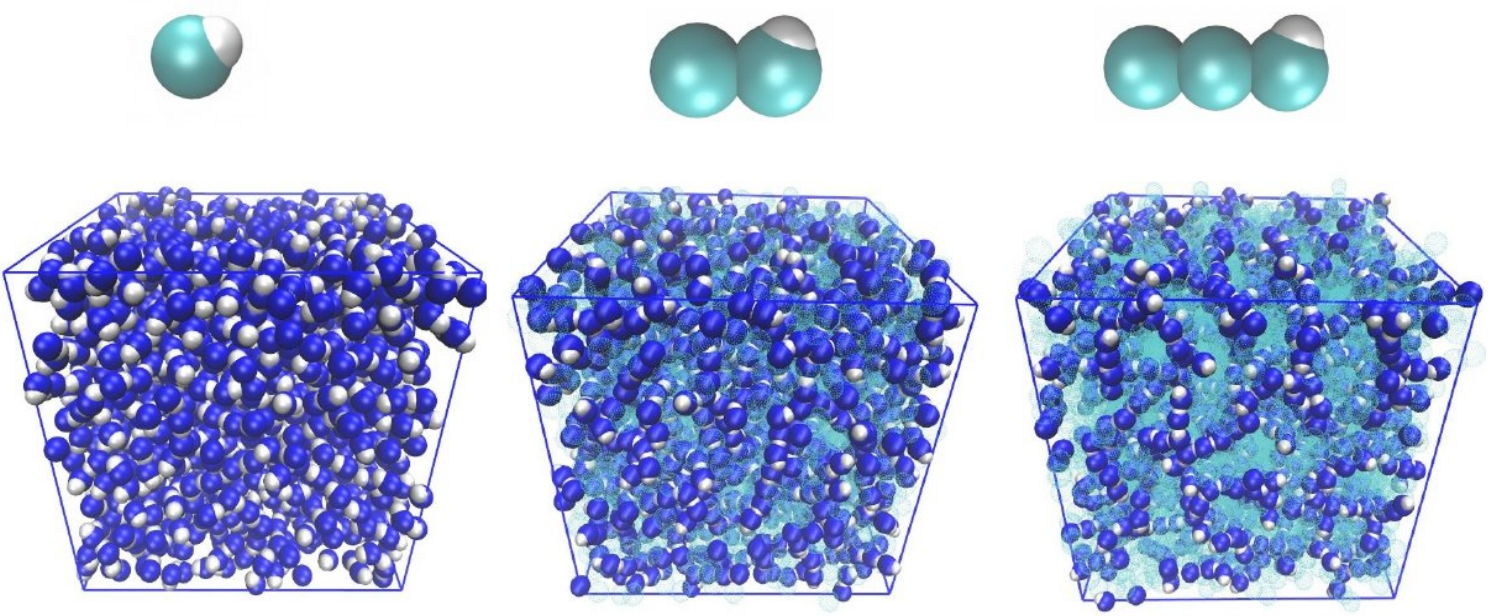
.Fig-8-Correlation functions (main panel) and structure factors (insets) of the model alcohols shown in Fig.7. $g_{--}$shown in blue, $g_{++}$in magenta and $g_{+-}$in green dashes (same conventions for the structure factors). The vertical lines in the insets indicate the main and BT peaks (yellow) and pre-peaks (orange) discussed in the text.

\section{Model 1}

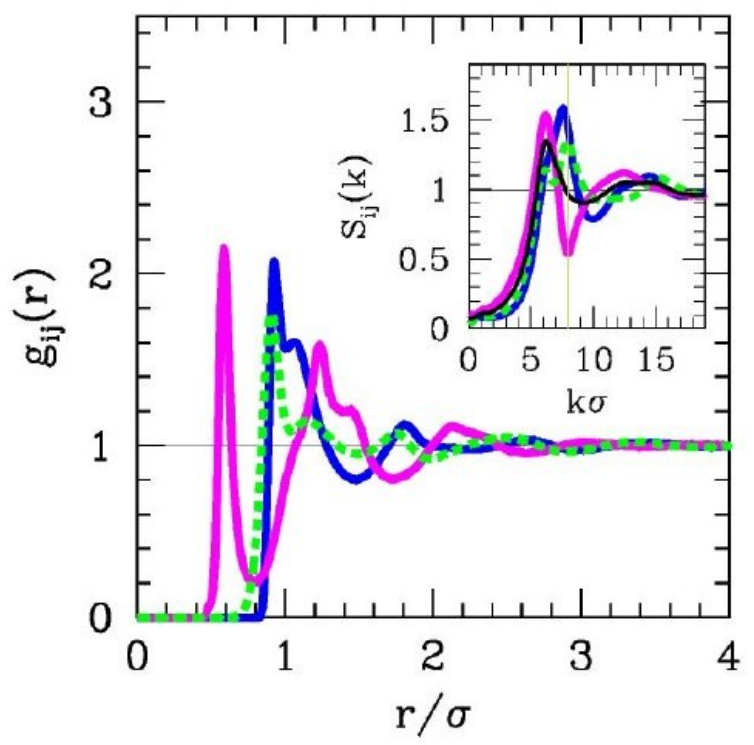

Model 2

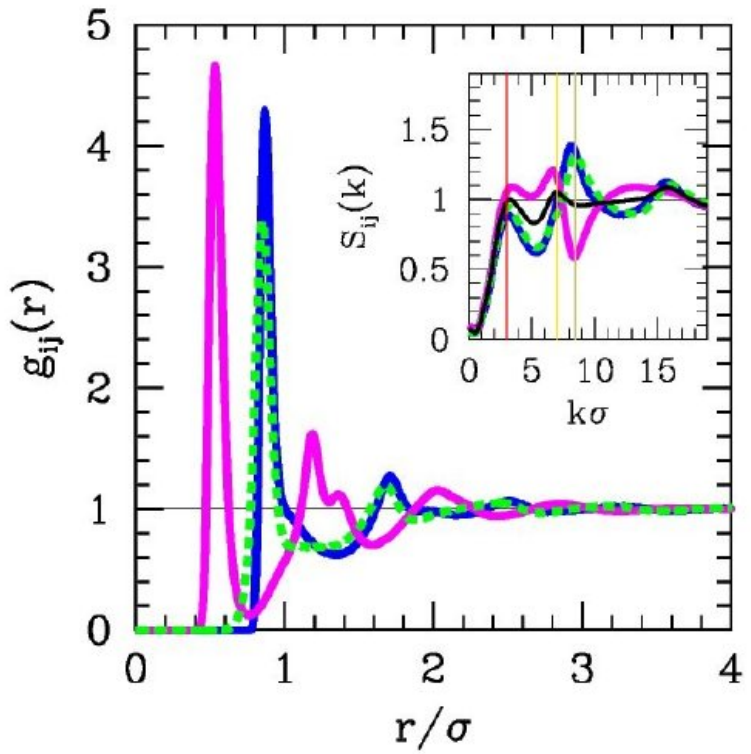


.Fig-9- Xray scattering functions for the model alcohols shown in Fig.7. Green lines for the ideal contribution and blue lines for total intensities. Full lines for model 1 , dotted for model 2 and dashed for model 3 , as labelled next to the appropriate lines

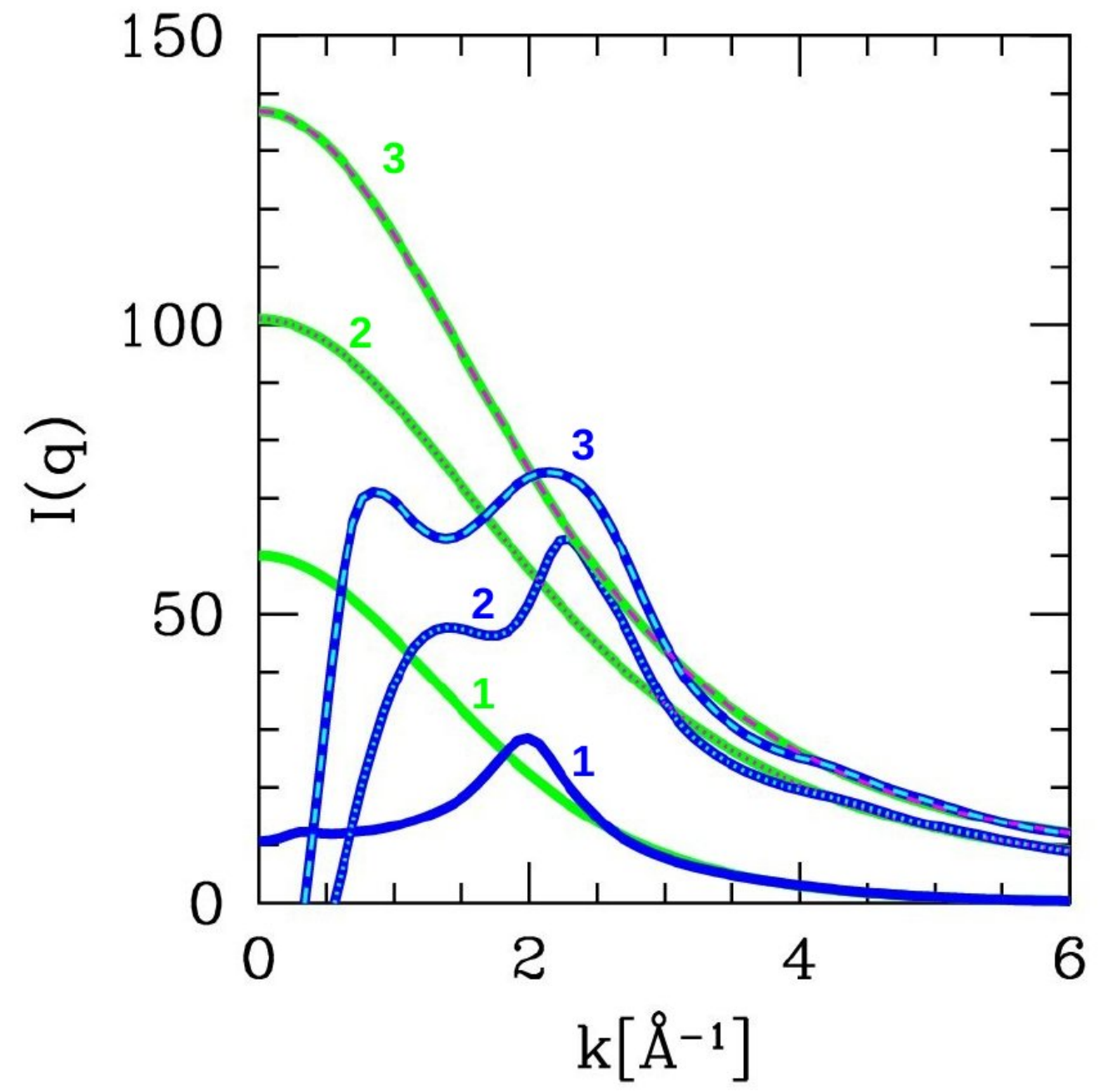


.Fig-10- Illustration of the origin of the pre-peak (see text) through transformation of the correlation function in real space (main panel) and Fourier space (inset). The original correlations are shown in magenta, the transformed one in blue, and the added Gaussian functions and their transforms in orange. The blue arrow in the inset shows the position of main peak and the red arrow the position of the pre-peak.

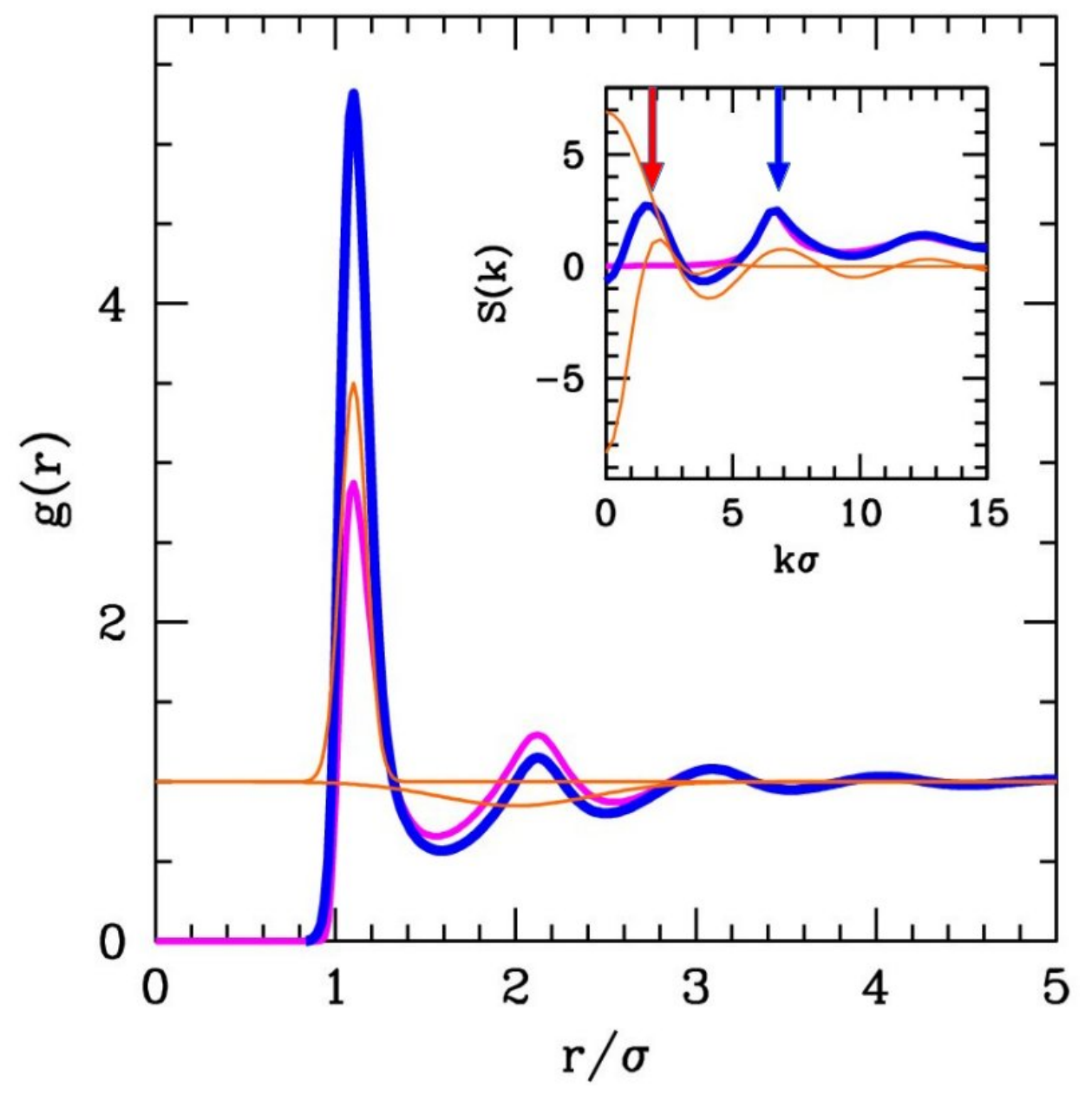

